\title{
Einige Versuche zur Biologie der Fischlarven.
}

Von

\author{
V. Franz (Helgoland).
}

Inhalt: 1. Das spezifische Gewicht der Fischlarven (S. 563). - 2. Osmotik der Fisch. larven (S. 567). - 3.! Reaktion lebender Fischlarven auf verschiedene Salzgehalte (S. 571). -

4. Phototropismus der Fischlarven (S. 577).

Das Folgende enthält einige der ersten und wie ich glaube, allgemein interessierenden Ergebnisse einer größeren Reihe von Untersuchungen, mit denen ich als Mitarbeiter bei der Internationalen Meeresforschung in der Kgl. Biologischen Anstalt auf Helgoland beschäftigt bin.

Seit der Begründung der „Internationalen Meeresforschung" steht die Erforschung der Wanderungen der Fische auf dem Programm dieser Organisation.

Man sprach im Anfang vom Problem der Wanderungen der Fische. Im Laufe der Jahre hat sich an den Gesichtspunkten der Forschung manches geändert, wie dies gewöhnlich der Fall ist, wenn Ergebnisse erzielt werden. Man erkannte, daß es nicht ein Problem der Fischwanderungen gibt, sondern daß hierin so viele Probleme verborgen sind, als es Arten von Fischen gibt. Jede Spezies muß für sich untersucht werden.

Die einschlägigen Untersuchungen sind nun begreiflicherweise noch lange nicht abgeschlossen, doch haben sie schon bei der einen Art mehr, bei der anderen weniger zu klaren, interessanten, überraschenden und teilweise rätselhaften Ergebnissen geführt.

Was die Fischbevölkerung der südöstlichen Nordsee betrifft, die ja von Helgoland aus ganz vorzugsweise erforscht wird, so ist hier der interessanteste Fisch augenscheinlich die Scholle (Pleuronectes platessa). Im allgemeinen kann man sagen: jede Größen- bzw. Altersstufe dieses Plattfisches beherrscht eine bestimmte Tiefe des Meeresbodens: im flachsten Wasser, am Strande in 0 bis $10 \mathrm{~m}$ Tiefe leben die jüngsten Stadien, der erste Jahrgang; und je älter sie werden, um so größere Tiefen suchen sie auf. Wie es scheint, ist diese allmähliche, seewärts gerichtete Wanderung keine gleichförmige, sondern sie wird alljährlich durch kürzere Rückwanderungen unterbrochen. Wenn die Fische die Geschlechtsreife er- 
langt haben, so haben sie meist bereits die 40-Meterlinie überschritten, und nur jenseits derselben, also in relativ tiefem Wasser, findet das Laichen statt. $^{1}$ )

Diese langsame, über Jahre ausgedehnte Wanderung kann man wohl beschreiben, aber viel schwieriger ist es, sie zu erklären. Ist es die Nahrungsfrage, die die Fische zum Wandern veranlaßt, wie dies besonders Heincke ${ }^{2}$ ) vermutet? Sind es Verhältnisse der Temperatur und des Salzgehaltes?

Noch schwerer verständlich ist uns die Larvenwanderung der Schollen. Die Eier werden ja über $40 \mathrm{~m}$ Tiefe abgelegt, d. h. rund 60 Seemeilen von der Küste entfernt, die Jungfischchen aber man findet nur unmittelbar an der Küste. Das ist nur dadurch möglich, daß die eben ausgeschlüften Larven sofort eine weite, nach der Küste gerichtete W anderung antreten, die sie in einigen Wochen vollenden. Man braucht diese Larvenwanderung übrigens nicht nur aus der Verbreitung der Eier einerseits, der Jungfische andererseits zu erschließen, man hat vielmehr in zahlreichen Planktonfängen bestimmte direkte Beweise dafür, daß sie stattfindet, wenn man auch nicht ganz genau weiß, ob z. B. die nordwestlich von Helgoland geborenen, oder aber die in der südlichen Nordsee geborenen Larven es sind, welche beispielsweise nach Helgoland gelangen. Man kann also auch die Larvenwanderung - in erster Näherung - beschreiben, hat aber wohl noch nie den Versuch gemacht oder angeregt, sie zu erklären.

Die Scholle ist nur das uns zunächst liegende Beispiel; auch andere Fische volltühren Wanderungen, und diese sind ebenso unerklärlich. Was treibt den Leptocephalus, vom Atlantischen Ozean, aus $1000 \mathrm{~m}$ Tiefe, nach den europäischen Küsten zu dringen, was treibt den jungen Glasaal flußaufwärts zu steigen?

Es harren also eine Unsumme von Fragen ihrer Lösung.

Dann und wann mögen Strömungen direkt eingreifen und Fischeier oder Larven gewisse Strecken weit transportieren, wie auch ähnliches von Damas $^{3}$ ) bezüglich des Calanus finmarchicus angenommen wird.

1) Heincke, F., Bericht über die Untersuchungen der Biologischen Anstalt auf Helgoland zur Naturgeschichte der Nutzfische. In: Die Beteiligung Deutschlands an der internationalen Meeresforschung. III. Jahresbericht, Berlin (Otto Salle) $190 \breve{5}$.

9) Vgl. Heincke, F., und Henking, H., Uber Schollen und Schollenfischerei in der südöstlichen Nordsee. In: Die Beteiligung Deutschlands an der Internationalen Meeresforschung, IV./V. Jahresbericht, Berlin (Otto Salle) 1908.

3) I) amas, D., Notes biologiques sur les copepodes de la mer du Nord. Publications de Circonstance, No. 22, Kopenhagen 1905. 
Aber im Falle des Leptocephalus ist ein direkter Transport kaum denkbar, und eigentlich ebenso scharf sprechen im Falle der Scholle viele Beobachtungen gegen eine derartige Annahme, z. B. die Beobachtung, daß Schollenlarven von dem holländischen Küstenmeere aus bis tief in die Zuidersee hineindringen. ${ }^{1}$ )

Es ist also kaum anders möglich, als daß die Wanderungen mindestens zum Teil aktiv erfolgen, die der Larven sowohl wie die der ausgewachsenen Fische.

Die Frage lautet also: Welche Reize wirken auf die Tiere ein? und: wie reagieren die Tiere auf die Reize?

Die Reize, an welche man denken könnte, sind etwa folgende: Hunger, Wärme und Kälte, Salzreichtum und Salzarmut sowohl als osmotische, wie als chemische Faktoren, Licht, hydrostatischer Druck, Strömung (letztere als Bewirkerin des "Rheotropismus"), endlich Tiefe als räumlicher Faktor.

Aus den Reaktionen auf solche Reize müssen die Wanderungsbewegungen in jedem Falle resultieren. Es bedarf keiner Erörterung, daß dieser Satz ganz unabhängig ist von der vielleicht gleichfalls sehr berechtigten Annahme, die Wanderungen seien "ererbte" Gewohnheiten, und daß er ihr nicht widerspricht; daß ferner das Problem sich nur unwesentlich verschiebt in jenen Fällen, wo statt des einzelnen Individuums ein ganzer Schwarm wandert, wo also die anführenden 'Tiere gewissermaßen den Kopf des Schwarmorganismus bilden.

Ich glaube bis jetzt, daß unter den genannten Reizen der Salzgehalt ein besonders wichtiger ist. Hierfür sprechen manche Beobachtungen. In der Nordsee, sowie in der Ostsee lajchen die Schollen an Stellen des relativ höchsten Salzgehaltes. Sehr ähnlich verhält sich die Flunder, ein Fisch, der sich biologisch von der Scholle hauptsächlich durch eine größere Tendenz zum brackigen und Süßwasser unterscheidet und dadurch die Abhängigkeit der Wanderungen vom Salzgehalt noch deutlicher verrät als die Scholle. Uberhaupt suchen viele Fische des Meeres zur Laichzeit ein salzreicheres Wasser auf als sonst (Kabeljau, Aal), genau umgekehrt verhalten sich der Stör, der Lachs, der Hering.

Ich habe daher bis jetzt hauptsächlich mit verschieden salzreichem Wasser experimentiert, um die Einwirkungen desselben auf die Tiere, bzw. die Reaktionen der Tiere auf jenes zu studieren.

Einen Faktor müssen wir wohl aus der Diskussion ausschalten, oder

1) Redeke, H. C. und van Breemen, P. J., Die Verbreitung der planktonischen Eier und Larven einiger Nutzfische in der südlichen Nordsee, mit einem Arihang über die Jungfische der Gadiden. Verhandl. nit het Rijksinstituut voor het Onderzoek der Zee, II. Deel, S'Gravenhage 1907-1908. 
doch sehr vorsichtig mit ihm umgehen: den Chemotropismus im gewöhnlichen Sinne. Wohl habe ich beobachtet, daB eine Centronotuslarve in einem Bassin, dessen Wasser an verschiedenen Stellen ungleichen Salz(gehalt hat, ${ }^{1}$ ) vor zu stark oder zu schwach salzigem Wasser ausweicht Aber im freien Meere handelt es sich um viel größere Dimensionen, so daß wir ein Eintreten dieser Reaktion kaum für Wanderungen verantwortlich machen können.

Ich hoffe, die Untersuchung auf eine breite Basis stellen zu können, so $\mathrm{da} B$ ich nicht nur mit Fischen, sondern auch mit anderen Tieren (Wvertebraten) arbeiten kann, da das Verständnis der Erscheinungen dadurch nur gefördert werden kann.

Spruchreife Ergebnisse habe ich jedoch bis jetzt weder bei Evertebraten, noch bei ausgebildeten Fischen in größerer Zahl zu verzeichnen, wohl aber bei Fischlarven.

Da die Versuche, obwohl nach Ausgangspunkt, Methode, Ziel und Ergebnissen durchaus unabhängig, doch eine gewisse Verwandtschaft haben mit solchen, wie sie heute von anderen: Loeb, Bethe, -- Sumner, Dakin - Wolfgang Ostwald - ausgeführt werden, so kann es nicht ohne Interesse sein, einige der bisherigen Ergebnisse zu veröffentlichen, zumal eine derartige Zusammenfassung den Autor selbst in seiner Arbeit zu fördern pflegt.

Ich glaube sagen zu dürfen, daß es sich um eine sehr aussichtsreiche Arbeit handelt. Denn auch so lange sie ihrem letzten Ziel, der Erkenntnis der Ursachen der Fischwanderungen, noch fern bleibt, führt uns jedes kleinste Resultat woiter in ein bisher vollständig unbekanntes Gebiet: die Biologie der Fischlarven. Das folgende mag es beweisen.

Um mit verschieden starken Seewasserlösungen arbeiten zu können, stellte ich mir zunächst durch Einkochen von gewöhnlichem Seewasser ein "eingedampftes Seewasser" her.

Den Salzgehalt der eingedampften Lösung habe ich nach Verdünnung auf $2 / 2$ aräometriert: Es betrug das spezifische Gewicht der auf $2 / 7$ verdünnten Lösung bei $17,5^{\circ} \mathrm{C} 1,02377$, woraus sich der Salzgehalt 31,13 ergibt. Mithin hat die eingedampfte Lösung das spezifische Gewicht 1,083195 (letzte Stelle rechnerisch unsicher) und den Salzgehalt 108,955\% (letzite Stelle dgl.).

Durch verschieden starkes Verdünnen mit destilliertem Wasser mittels Pipette konnte ich mir hieraus 21 verschieden starke Seewasserlösungen herstellen, und zwar in folgender Weise (Tabelle I).

1) Für kurze Zeit läbt sich dieser Zustand bei sehr flachem Wasser herstellen. 
Tabelle I.

\begin{tabular}{|c|c|c|c|c|}
\hline $\mathrm{Nr}$. & 1 & 2 & 3 & 4 \\
\hline Teile der eingedampften Lösung & 0 & $0, \overline{5}$ & 1 & 1,5 \\
\hline Teile $\mathrm{H}_{2} \mathrm{O}$ & 10 & $9, \breve{5}$ & 9 & 8,5 \\
\hline $\begin{array}{l}\text { Spezifisches Gewicht des Ge- } \\
\text { misches bei } 17,5^{\circ} \mathrm{C} \ldots \ldots \ldots\end{array}$ & 1,0000000 & 1,00416 & 1,00832 & 1,01248 \\
\hline Konzentration d. Gemisches in $\%$ & $\mathbf{0}$ & 5,45 & 10,90 & 16,34 \\
\hline
\end{tabular}

\begin{tabular}{|c|c|c|c|c|c|}
\hline 5 & 6 & 7 & 8 & 9 & 10 \\
\hline 2 & 2,5 & 3 & $3, \check{o}$ & 4 & 4,5 \\
\hline 8 & $7, \tilde{\jmath}$ & 7 & 6,5 & 6 & $5, \check{5}$ \\
\hline 1,01664 & 1,02080 & 1,02496 & 1,02912 & 1,03328 & 1,03744 \\
\hline 21,79 & 27,24 & 32,69 & 38,13 & 43,58 & 51,03 \\
\hline 11 & 12 & 13 & 14 & 15 & 16 \\
\hline$\tilde{5}$ & $\check{\partial}, \check{\partial}$ & 6 & 6,5 & 7 & 7,5 \\
\hline $\bar{j}$ & $4, \tilde{o}$ & 4 & 3,5 & 3 & 2,5 \\
\hline 1,04160 & 1,04576 & 1,04992 & 1,05408 & $1,05 \overline{824}$ & 1,06240 \\
\hline 56,48 & 61,92 & 67,37 & 72,82 & 77,27 & $\$ 2,72$ \\
\hline
\end{tabular}

\begin{tabular}{c|c|c|c|c}
\hline \hline 17 & 18 & 19 & 20 & 21 \\
\hline 8 & 8,5 & 9 & 9,5 & 10 \\
2 & 1,5 & 1 & 0,5 & 0 \\
1,06656 & 1,07072 & 1,07487 & 1,07903 & 1,0832 \\
$\mathbf{8 7 , 1 6}$ & $\mathbf{9 2 , 6 1}$ & $\mathbf{9 8 , 0 6}$ & $\mathbf{1 0 3 , 5 1}$ & $\mathbf{1 0 8 , 9}$
\end{tabular}

Für die meisten Versuche stellte ich mir die Gemische aus der eingedampften Lösung neu her und zwar mit den Mengen, welche zusammen je $100 \mathrm{ccm}$ ergaben, z. B. Lösung 7 aus $30 \mathrm{~cm}$ der eingedampften Lösung und $70 \mathrm{ccm} \mathrm{H}_{2} \mathrm{O}$. Die $100 \mathrm{ccm}$ füllten dann meist ein Wasserglas etwa zur Hälfte.

Es hat sich gezeigt, daß ein oder selbst mehrere kleine Tiere bei der Versuchsanordnung nicht unter Sauerstoffmangel litten, vielmehr tage- 
und wochenlang darin leben konnten und sicher nur aus anderen Gründen schließlich absterben.

Uber die Zuverlässigkeit der Bestimmungen kam ich zu einem recht befriedigenden Urteil. Hergestellt und bestimmt habe ich die eingedampfte Lösung am 9. November 1908. Die hier zu besprechenden Versuche fielen dann meist in die Monate Januar bis April 1909. Am 21. Mai 1909 habe ich Lösung 7 nochmals gemischt und aräometriert. Ich fand spezifisches Gewicht bei $17,5^{\circ} \mathrm{C} 1,002500$ und Salzgehalt $32,74 \%$. Die Abweichung gegen die frühere, vor einem halben Jahre ausgeführte Bestimmung beläuft sich also auf 0,000004 im spezifischen Gewicht und $0,05 \%$ im Salzgehalt. Herr Kollege Dr. Reichart führte auf meine Bitte eine Titration aus, sie ergab 32,77\%, also wiederum eine durchaus befriedigende Ubereinstimmung. Die ganz geringfügigen Abweichungen machen bei meinen Versuchen garnichts aus.

Dennoch will ich nicht unterlassen, der peinlichen Genauigkeit halber darauf hinzuweisen, daß die Zahlen des spezifischen Gewichts und Salzgehaltes nach Bestimmung der einen Lösung rechnerisch gefunden sind unter der Annahme, daß beim Mischen mit destilliertem Wasser keine Volumänderung eintritt. Diese Annahme dürfte kaum ganz zutreffen, vielmehr eine minimale Kondensation eintreten, die jedoch eine Ẍnderung unserer Zahlen frühestens in der zweiten Dezimalen zur Folge hätte und sich daher im Ausfall unserer Ergebnisse garnicht bemerkbar machen kann.

Auf noch einen Punkt sei hingewiesen, der vielleicht von irgend einer Seite beanstandet werden könnte. Schon wenn man Seewasser auf etwa die Hälfte des Volumen eindampft, fällt eine geringe Spur eines Salzes (CaCO ${ }_{3}$ ?) aus. Dieser Lmstand kann weder für das spezifische Gewicht, noch für den Salzgehalt, noch für den osmotischen Druck der Gemische irgendwie in Frage kommen, wie die Übereinstimmung zwischen Aräometrierung und Filtration beweist. Denkbar wäre höchstens, daß eine Verminderung der Metalloder der Hydroxyl-Ionen die Tiere in irgend einer Weise chemisch affizierte. Hiergegen habe ich jedoch $\mathrm{zu}$ bemerken, daß die Versuchstiere in Lösung 7, die ja mit 32,7\% als ein für Helgoland normales Seewasser gelten kann, niemals die geringste Abweichung vom normalen Verhalten verrieten. Man kann also ruhig sagen, daß sich der verminderte Gehalt eines bestimmten Salzes in keiner Weise bemerkbar machte.

Immerhin werde ich vielleicht in Zukunft vorzugsweise nur mit solchen Lösungen arbeiten, die ich mir durch Verdünnen von natürlichem Seewasser direkt herstelle, denn es hat sich gezeigt, daß die Abstufungen zwisehen 0 und $33 \%$ zu viel interessanteren Ergebnissen führen als diø höheren Salzgehalte, welchen ja auch die Tiere in der Natur niemals ausgesetzt sind. 
Uberhaupt kamen Lösung 18 bis 21 in keinem Falle zur Verwendung; dagegen wurde in manchen Fällen die Zahl der Abstufungen noch vergrößert.

\section{Das spezifische Gewicht der Fischlarven.}

Die allereinfachste und erste Erscheinung, welche bei den Versuchen auffällt, ist, daß die Fischlarven in den stärkeren Lösungen oben auf schwimmen, in den stärkeren aber untersinken.

Es zeigte sich auch sehr bald, daß die verschiedenen Spezies sich hierin verschieden verhalten.

Meines Wissens ist die Frage nach dem verschiedenen spezifischen Gewicht der Fischlarven noch niemals erörtert worden, obwohl vielleicht schon manchem aufgefallen ist, daB z. B. die Centronotus-Larve nur am Boden eines GefäBes ruhen kann, die Schollenlarve an der Oberfläche.

Freilich ist schon diese Beobachtung mit aller Art Schwierigkeiten verbunden: 1. man muB möglichst absolut frisches Material haben; geschädigte Larven pflegen immer unterzusinken. 2. Die Tiere können, wenn einmal mit Luft in Berührung gebracht, an der Wasseroberfläche von den "Oberflächenhäutchen" getragen werden; wenigstens scheint mir diese Erklärung anzunehmen zu sein für die zahlreichen Fälle, wo Larven an der Oberfläche von Flüssigkeiten hafteten, für die sie viel zu. schwer waren. 3. Sobald sich die Larve aktir bewegt, kann man ihr spezifisches Gewicht aus nichts mehr erschließen.

Um nun möglichst exakt das spezifische Gewicht für jede Spezies zu bestimmen, mußte ich die genannten Schwierigkeiten überwinden.

Offenbar war schon viel gewonnen, wemn es gelang, die Tiere zu immobilisieren.

Zu diesem Zwecke durfte ich sie nicht töten, denn tut man dies, sei es durch Druck, sei es durch Ersticken, so sinken sie unfehlbar auf den Grund, auch bei der stärksten von mir angewandten Lösung.

Ich konnte aber die Larven im ausgehöhlten Objektträger, unter dem Deckglas, durch Luftabschlub betäuben und mußte dann natürlich den geeigneten Moment abpassen, in welchem die Körpermuskulatur außer Tätigkeit gesetzt, das Herz aber noch in reger Pulsation begriffen ist. In diesem Moment ist die Larve zum Versuch geeignet, man kann sie mit einer Pipette in die Lösung bringen.

Ubrigens empfiehlt sich im vorliegenden Falle, statt die oben erwähnten Wassergläser zu benützen, eine abweichende Methode, die hierbei auch dann zum Ziele führt, wenn nur wenige Larvenindividuen vorliegen.

Ich schichtete nämlich die verschieden starken Salzlösungen in einem weiten, hohen Meßzylinder vorsichtig übereinander, die schwerste zu unterst. Durch das Phänomen der Schlierenbildung hat man die genügende Kontrole 
darüber, ob die Schichtung gelingt. Nötigenfalls nimmt man größere Mengen jeder Lösung oder einen engeren Zylinder. Ich benutzte meist Meßzylinder für 500 oder für $1000 \mathrm{ccm}$ und nahm von jeder Lösung $100 \mathrm{ccm}$.

Bringe ich alsdann eine Larve mittels Pipette in die zu oberst liegende Schicht, so sinkt sie bis in diejenige Tiefe, wo das Wasser ihr spezifisches Gewicht hat. Und zwar sinkt sie zunächst schnell, dann langsamer kopfab, in der betreffenden Schicht aber legt sie sich horizontal. Man kann dann also mit einer Genauigkeit, welche durch den Abstufungsgrad der verschiedenen Lösungen gegeben ist, das spezifische Gewicht der Larve ablesen.

Die Ergebnisse lassen sich am besten schematisch darstellen.

Tabelle II.

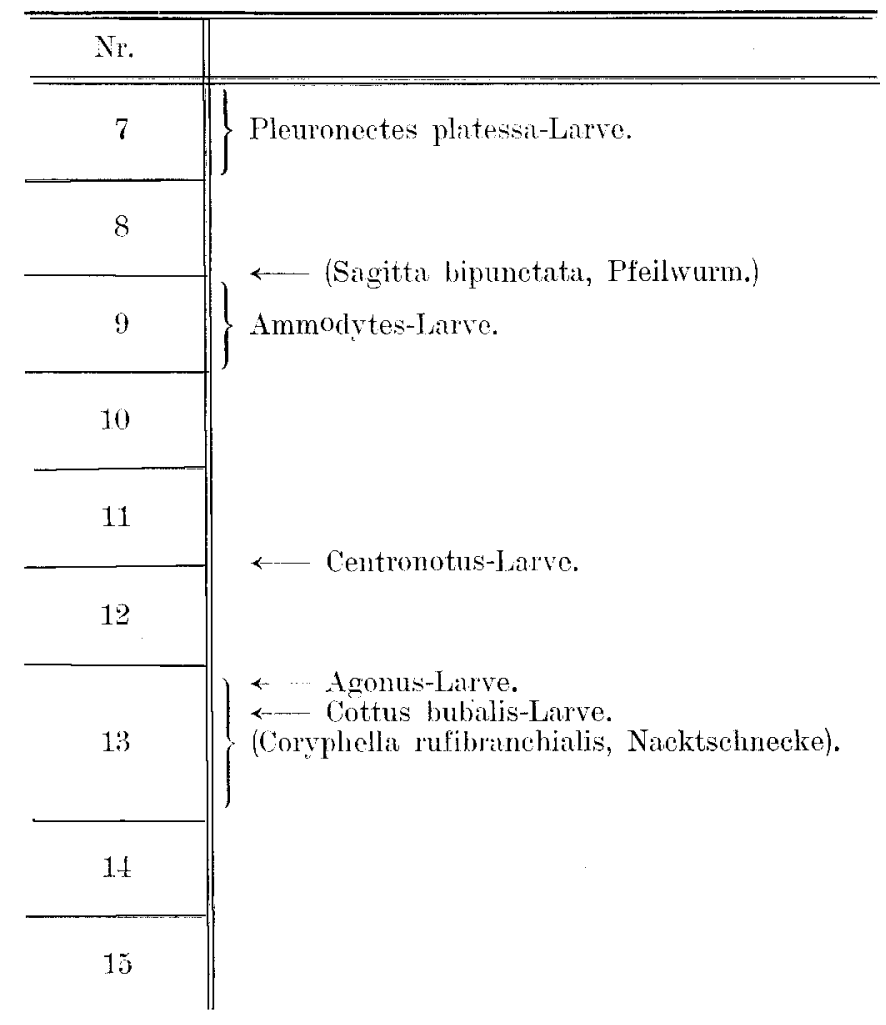

$\mathrm{Zu}$ dieser schematischen Darstellung ist im einzelnen folgendes $\mathrm{zu}$ bemerken:

Daß die Pleuronectes platessa-(Schollen-)Larve ${ }^{1}$ ) das relativ geringe Gewicht der Lösung 7 hat, ist bis jetzt nur aus Beobachtungen in den

1) Die Eier waren planktonisch gefischt, die Larven im Laboratorium ausgeschlüpft. 
Wassergläsern - nicht in dem Meßzylinder - zu entnehmen. Da diese Beobachtungen aus den erwähnten Gründen nicht ganz einwandfrei sind, so bedarf das Ergebnis noch der Erhärtung.

Sicher ist, daß die Ammodytes-Larve ${ }^{1}$ ) in ihrem spezifischen Gewicht etwa einem Seewasser von $43,6 \%$ gleichkommt und mithin etwa 1,033 beträgt. Der Versuch wurde mit 10 Larven auf einmal ausgeführt, die übereinstimmend das erwähnte Resultat ergaben.

Um ein weniges leichter fand ich den Pfeilwurm, Sagitta. $\left.{ }^{2}\right)$ Doch bedarf auch dies Ergebnis noch der Erhärtung.

Bedeutend schwerer als Seewasser sind dagegen die Larven von Centronotus, Agonus und Cottus. ${ }^{3}$ ) Für Centronotus und Agonus ist das Ergebnis durchaus einwandfrei, für Cottus bubalis liegen nur drei voneinander ziemlich abweichende Beobachtungen vor: die eine Larve schwebte an der Grenze von Lösung 12 gegen 13, die zweite 11 gegen 12, die dritte 14 gegen 15. Sucht man einen Mittelwert zu gewinnen, so fällt er mit dem für die Agonuslarve gewonnenen etwa zusammen.

Das spezifische Gewicht der, uns als planktonisch bekannten. Fischlarven variiert also im hohen Grade nach der Spezies.

Und es ist wohl bemerkenswert, daß eine Agonuslarve kaum mehr hinter der Nacktschnecke Coryphella rufibranchalis zurücksteht. Coryphella ist, wie alle Schrecken, kein Planktont, sondern ein Bodentier, wenn auch nicht eins der schwersten: Es pflegt auf Hydroidpolypenstöcken umberzuklettern, ohne daß diese zusammenbrechen.

Nach diesen Ergebnissen liegt schon die Annahme außerordentlich nahe, dab die Centronotus-, Agonus- und Cottuslarve gleichfalls bis zu gewissem Grade Bodentiere sind, d. h. daß sie vermöge ihres höheren spezifischen Gewichts in höherem Grade an den Boden gebunden sind, als die Platessa-, Ammodyteslarve und sicher sehr viele andere Fischlarven und überhaupt Planktontiere. Die Annahme wird weiter dadurch wahrscheinlich, daß wenigstens die Agonus- und Cottuslarven lebhaftest gefürbt sind im Gegensatze zur Mehrzahl der Planktontiere; endlich dadurch, daß bei den genannten Arten auch das Ei wie der ausgebildete Fisch am Grunde leben.

Ich möchte für die Gesamtheit der Organismen, die zwar nicht echte Bodentiere, aber auch nicht echte pelagische Tiere sind, den Namen

$$
\text { benthopelagisch }
$$

1) Die Larven waren planktonisch gefischt.

9) Planktonisch gefischt.

3) Die Centronotuslarven waren als Eierballen vom Grunde gefischt und schlüpten, noch während sie an die Oberfläche geiracht wurden, aus. Die Agonus- und Cottuslarven waren planktonisch gefischt. 
vorschlagen, der in gewissen Fällen vielleicht durch benthoplanktonisch $\mathrm{zu}$ ersetzen wäre. (Zum Plankton im strengsten Sinne - zum "Treibenden " gehören allerdings eigentlich nur Eier, Cysten und Pflanzen; erweitert man den Begriff auf die Tiere mit Eigenbewegung, so wird er in jedem Falle unscharf, und dann werden wir ihn am besten durch pelagisch ersetzen.) Das Wort Benthopelagial ist seinem Inhalte nach natürlich weit verschieden von Bathypelagial und bezeichnet eigentlich nur eine $\mathrm{Zw}$ ischenstufe zwischen Pelagial und Benthos; es verdient aber verwendet zu werden, weil wahrscheinlich zu dieser Zwischenstufe eine bemerkenswerte, große Zahl von Tieren gehört.

Zum Benthopelagial gehören z. B. von den Fischen die große Gruppe der Gadiden: Es sind pelagische Fische, doch suchen sie ihre Nahrung ganz oder teilweise am Grunde, und sie zeigen deutliche Anpassungen ans Leben am Grunde: Ausbildung von Bartfäden, Umwandlung von Flossenstrahlen zu unverzweigten, spitz auslaufenden Tastern. Und von den Fischlarven sind bis jetzt die genannten drei Arten dem Benthopelagial zuzurechnen.

Es ist dies ein Ergebnis, das durch keine Planktonfischerei mit den bis jetzt gebräuchlichen Methoden zu erlangen war, weil ja die Planktonfischerei in der Nähe des Grundes immer untunlich wird. Die Planktonfischerei konnte vielmehr nur zu dem Ergebnis führen, daß jene Larven im Plankton vorkommen.

Nicht immer gehören Ei, Larve und Jungfisch derselben Lebensgemeinschaft an, vielmehr gibt es manchen interessanten Wechsel, wie schon die folgende kleine Tabelle lehrt (Tabelle III).

Tabelle III.

\begin{tabular}{|c|c|c|c|}
\hline Spezies & Ei & Larve & Jungfisch \\
\hline Pleuronectos platessa ... & pelagisch & pelagisch & benthonisch \\
\hline Ammodytes ........ & benthoniseh & $"$ & benthopelagisch \\
\hline Centronotus........ & $\because$ & benthopelagisch & benthoniseh \\
\hline Agonus $\ldots \ldots \ldots$ & . & $"$ & $"$ \\
\hline 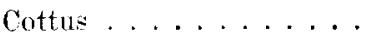 & . & " & $"$ \\
\hline Gudus morrhua. . . . . . & pelagisch & (pelagisch?) & benthopelagisch \\
\hline Clupen harengus . . . . . & benthonisch & (pelagisch?) & pelagisch \\
\hline Glupea sprattus ..... & pelagisch & (pelagiseh?) & $"$ \\
\hline
\end{tabular}

Es ist ein „kaleidoskopartig buntes Bild", um diesen Ausdruck zu gebrauchen, den Henking für die vielen spezifischen Verschiedenheiten im. Modus der Wanderungen der Fische fand. 


\section{Osmotik der Fischlarven.}

Es ist seit etwa 40 Jahren bekannt, daß die Knochenfische, im bedeutungsvollen Gegensatze zu den Selachiern und zu den Evertebraten; Körperflüssigkeiten (Blut, Lymphe) von sehr geringem osmotischem Druck und Salzgehalt haben. Bei Selachiern und Evertebraten sind dagegen die Körperflüssigkeiten von gleichem osmotischem Drucke wie das umgebende Seewasser, und bei Evertebraten kommt auch der Salzgehalt des "Milieu extérieur" dem des "Milieu intérieur" gleich. Diese beiden physiologischen Tiergruppen sind auch hinsichtlich des osmotischen Druckes ihrer Flüssigkeiten in hohem Grade von den Schwankungen des Salzgehaltes des Meeres abhängig; die Knorpelfische stehen dagegen viel unabhängiger, wenn auch [Sumner, $\left.{ }^{1}\right)$ Dakin $\left.\left.{ }^{2}\right)\right]$ nicht ganz unabhängig da.

Wie verhalten sich die Larven der Knochenfische?

Sieht man von den Selachiern ab, bei denen ja besondere Verhältnisse herrschen, so könnte der Gegensatz zwischen Teleostiern und Evertebraten wohl durch die viel dickeren Körpermembranen der ersteren erklärt erscheinen. $\mathrm{Zu}$ meiner Überraschung erfuhr ich jedoch durch meine Beobachtungen an Fischlarven, daß dieser Schlub irrig wäre. Auch die Larven der Fische stehen, obwohl sie äußerst zarte Tierchen sind, schon osmotisch unabhängig da.

Eine Fischlarve mag nämlich viele Tage lang in ganz geringem, für sie entschieden abnormen Salzgehalte, z. B. 5 bis $10 \%$, oder in erhöhtem $(40 \%)$ leben, sie wird nicht die geringste Quellung oder Schrumpfung erkennen lassen.

Ganz anders verhalten sich Evertebraten. Trochus zizyphinus, eine Gehäuseschnecke, fühlte sich offenbar in Lösung 7 am wohlsten, ihr Verhalten war hier ein durchaus normales. In stärkerem (Lösung 8 bis 12) wie in schwächerem Salzgehalt (1 bis 6) hatte sie das Vermögen, aus dem Gehäuse hervor- und im Aquarium umher zu kriechen, sichtlich verloren und zwar genau stufenweise in um so stärkerem Maße, je mehr die Lösung von der Norm abwich. Daß hierbei die osmotischen Verhältnisse eine wesentliche, wenn auch vielleicht nicht ausschließliche Rolle spielen, wird durch das stark kontrahierte, man darf wohl sagen geschrumpfte Aussehen der Tiere in den starken Lösungen und die vollkommen, durch 12 stündigen Aufenthalt in normalem Seewasser erreichte Reversibilität der Wirkung wahrscheinlich, wenn auch nicht gewib: denn es ist schwer, Kontraktion und Schrumpfung genau zu unterscheiden, auch muß ich be-

1) Sumner, F. B., Physiological effects upon fishes of changes in density and salinity of water. Bull. U. S. Bureau of Fisheries. 1905.

2) Dakin, W. J., The osmotic concentration of the blood of fishes taken from. sea water of naturally varying concentration. Bio-Chemical Journal 1907, Vol. III. 
merken, daß die Reversion in Lösung 3 bis 6 und 10 sehr sehnell - bei der Langsamkeit der Schneckenbewegung kann man sagen sofort - begann. Einwandfreier ist die Beobachtung, daß Vorticella campanula, dem SüBwasser entnommen, in Salzwasser sich verkleinert und stark runzelig wird, also ganz offenbar durch Wasserverlust schrumpft. (Die Notizen darüber habe ich seit mehreren Jahren). Auch diese Wirkung ist reversibel, wenn sie nicht zu lange anhält. (Echinodermen und Crustaceen liefern begreiflicherweise keine so klaren Resultate; daß aber Wirbellose überhaupt in die Rahmen der Untersuchung gezogen werden müssen, mögen die erwähnten, eben leider noch spärlichen Beobachtungen zeigen.)

Die Fischlarve schrumpft oder quillt erst auf, wenn sie tot ist. Ich habe, nachdem ich dies erkannt, bei allen Fischlarven, die infolge Hungers oder infolge der dauernden Einwirkung abnormer Lösungen abstarben, den Zustand der abgestorbenen Larve untersucht. Quellung, das Anzeichen, dab sie in einem hypotonischen Seewasser lebte, ist sehr leicht am ganzen Körper zu erkennen, ferner, wenn ein Dottersack vorhanden ist, daran, daß er prall gefüllt ist und hierdurch eine Rückwärtskrümmung der Wirbelsäule erzwingt. Schrumpfung ist namentlich leicht am Flossensaum erkennbar, der sich in Falten legt, ferner wiederum am Dottersack, der sich in Falten legt und in diesem Zustande eine Vorwärtskrümmung der Wirbelsäule erzwingt. (Mit Vorsicht ist das Auge zu verwerten. Das Hornhautepithel, das im Leben einen osmotischen Stoffaustausch verhindert, ${ }^{1}$ ) wird zwar post mortem diese Fähigkeit verlieren. Ist das Auge prall gefüllt, so kann man daher Quellung annehmen. Ist es aber eingesunken, so ist das kein Beweis einer osmotischen Schrumpfung. Es sinkt auch bei noch lebenden, aber moribunden Larven manchmal ein, und zwar auch in schwachem Salzgehalt, wahrscheinlich infolge des Nachlassens des arteriellen Druckes). Wo weder Schrumpfung noch Quellung erfolgt, ist der osmotische Druck des Blutes dem des Meerwassers isotonisch. Die folgende Tabelle IV zeigt, wo dieser Punkt bei den verschiedenen Spezies liegt.

Für Pleuronectes platessa habe ich noch eine Versuchsreihe aufzuweisen, bei der ich mit Lösung Nr. 6 bis 8 operierte, die Zahl der Abstufungen jedoch verdoppelte, so daß ich zwischen Nr. 6 und̀ ? eine Nr. 6,5 einschiebe, und zwischen Nr. 7 und 8 eine 7,5 (s. Tabelle V, S. 570 \%

Für Pleuronectes platessa lag in Tabelle IV nur je 1 Individuum vor; hiernach schien diese Spezies hinsichtlich ihres osmotischen Druckes einem Seewasser, das zwischen Lösung 6 und 7. und näher der ersteren steht, zu gleichen. Tabelle $\mathrm{V}$ zeigt aber, wie sich das Bild verändert, und wie

1) V. Franz, Beobachtungen am lebenden Selachierauge. Jenaische Zeitschr. 1906, Bd. XLI. 


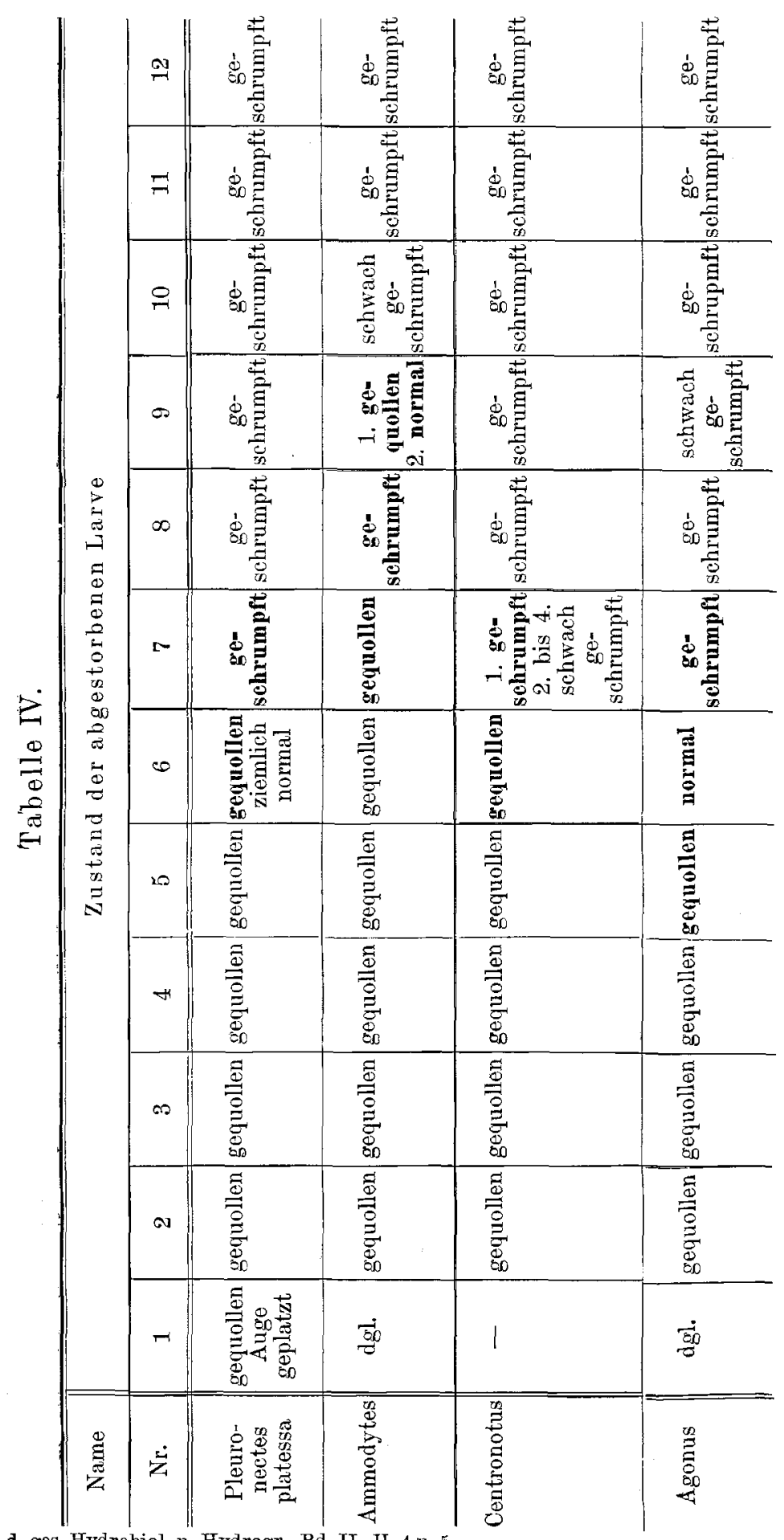

Revre d. ges. Hydrobiol, u. Hydrogr. Bd, II. H, 4 n, 5. 
Tabelle V.

\begin{tabular}{|c|c|c|c|c|c|}
\hline Name & & Zustand d & der abgestorben & Ien Larve & \\
\hline Nr. & 6 & 6,5 & 7 & 7,5 & 8 \\
\hline $\begin{array}{l}\text { Pleuronectes } \\
\text { platessa }\end{array}$ & $\mid \begin{array}{ll}\text { 1. } & \text { normal } \\
\text { 2. } & \text { geschrumpft }\end{array}$ & $\begin{array}{l}\text { 1. geschrumpft } \\
\text { 2. u. 3. schwach } \\
\text { geschrumpft }\end{array}$ & $\begin{array}{l}\text { 1. u. 2. schwach } \\
\text { geschrumpft }\end{array}$ & $\begin{array}{l}\text { 1. normal } \\
\text { 2. gequollen } \\
\text { 3. und 4. ge- } \\
\text { geschrumpft }\end{array}$ & $\begin{array}{l}\text { 1. normal } \\
\text { 2. schwach ge- } \\
\text { schrumptt } \\
\text { 3. geschrumpft }\end{array}$ \\
\hline
\end{tabular}

es infolge individueller Variationen unschärfer wird, wenn man die Zahl der Versuchstiere vergrößert.

Die Angaben für Centronotus beziehen sich überall auf mehrere Individuen - die Zahlen habe ich nicht notiert --, sie können als gut gesichert gelten.

Dagegen liegen für Ammodytes und Agonus meist nur je 1 Exemplar vor, die Angaben verdienen daher noch präzisiert zu werden.

Immerhin ist deutlich erkennbar, daß der osmotische Binnendruck der Larven ein bestimmter ist - anscheinend etwa dem des Seewassers von $33,3 \%$ gleichend - und daß er auch durch tage- oder wochenlanges. Verweilen der lebenden Tiere im hypotonischen oder hypertonischen Seewasser nicht geändert wird.

Geradezu verwüstende Wirkungen hat ferner das destillierte Wasser. Ihm scheint auch eine physikalisch-chemische Wirkung auf den lebenden Körper zuzukommen, wobei die besondere Schädlichkeit des Fehlens aller Salze übrigens nichts noues wäre. Im destillierten Wasser lebt eine Larve keine sechs Stunden - oft nur eine halbe - sie springt ruhelos hin und her, bis sie ermattet hinfällt, um vielleicht noch einige Male aufzuspringen. Untersucht man den abgestorbenen Körper, so findet man fast immer das Auge geplatzt, so daß das schwarze Augenpigment unter der Conjunctiva versprengt ist, oder, wenn auch diese geplatzt ist, heraustritt. Das Auge mag wie nach Dakin ${ }^{1}$ ) beim Hai, einen besonders hohen osmotischen Druck in seinem Inneren haben und hat daher in so starkem Maße unter dem destillierten Wasser zu leiden. Da all dieses in Lösung 2 $(4,2 \%$ nicht mehr einzutreten pflegt, so habe ich gelegentlich noch vier Zwischenstufen zwischen Lösung 1 und 2 eingeschaltet und in jeder das Verhalten der Schollenlarven geprüft. Jede Larve wurde hierbei zunächst in ein Glas mit der gewünschten Lösung, dann von hier aus in ein zweites mit derselben Lösung gebracht, um die Lösung ganz

1) Dakin, W. J., Variations in the osmotic concentration of the blood and osmotic fluids of aquatic animals, caused by changes in the external medium. Bio-Chemical. Journal 1908, Vol. III. 
frei von ungewollten Beimischungen zu erhalten. Es zeigte sich, daB in Lösung 1,2 (d. i. 0,1 Teile eingedampftes Seewasser, 9,9 Teile $\mathrm{H}_{2} \mathrm{O}$, Konzentration des Gemisches $=1,1 \%$ der Tod zwar auch schon nach vier Stunden eingetreten war, doch nicht unter so starken Quellungserscheinungen. In Lösung 1,4 (Konzentration 2,2\%) trat der Tod erst am vierten Tage ein, noch später bei $3,3,4,4$ und $5,6 \%$.

Der Unterschied zwischen der verwüstenden Wirkung des gänzlich salzfreien Wassers und dem schon sehr starken lebenerhaltenden Einfluß der geringsten Spuren Salzgehaltes ist jedenfalls wohl eine Erscheinung, die sich rein osmotisch nicht verstehen läßt.

\section{Reaktion der lebenden Fischlarven auf verschiedene Salzgehalte.}

Wir kommen zum wichtigsten Teile, zu der Frage, mit welchen Lebensäußerungen die Tiere - in unserem Falle die Fischlarven - auf verschiedene Salzgehalte reagieren.

Ich habe das Verhalten jedes einzelnen Versuchstieres längere Zeit hindurch $^{1}$ ) fast täglich einmal geprüft und mir Notizen wie: lebt, munter, normal, lebhaft, zappelt, träge, lahm, regungslos, tot usw. gemacht.

Es wird aber nicht nur der Vereinfachung, sondern auch der Erkenntnis des Prinzipiellen dienen, wonn ich in den folgenden Tabellen den Zustand der lebenden Larve kurz als erregt (r), normal (n) oder gelähmt (I) bezeichne.

VerhältnismäBig arm an auffälligen Ergebnissen war die Versuchsreihe mit Ammodytes (Tabelle VI).

Tabelle VI.

Ammodyteslarve. Beginn des Versuches 3. Hebruar 1909.

\begin{tabular}{|c|c|c|c|c|c|c|c|c|c|c|c|c|}
\hline & & 1 & 2 & 3 & 4 & 5 & 6 & 7 & 8 & 9 & 10 & 11 \\
\hline \multicolumn{2}{|c|}{ am 3. Versuchstage } & tot & $\mathrm{n}$ & $\mathrm{n}$ & 1 & $\mathrm{n}$ & n & tot & Il & $n$ & tot & $\mathbf{r}$ \\
\hline$" 4$. & $"$ & - & $\mathrm{n}$ & $n$ & tot & $n$ & $n$ & - & $n$ & $n$ & - & tot \\
\hline "5. bis 8. & $"$ & - & $\mathrm{n}$ & $\mathrm{n}$ & - & $\mathrm{n}$ & $n$ & - & n & $\mathrm{n}$ & - & - \\
\hline$" 9$ & $"$ & - & n & $n$ & -- & $\mathrm{n}$ & $\mathrm{n}$ & - & $\mathrm{n}$ & tot & - & - \\
\hline "10. bis 13. & $"$ & - & $\mathbf{n}$ & $\mathrm{n}$ & -- & $\mathrm{n}$ & $\mathrm{n}$ & 一 & n & 一 & - & 一 \\
\hline$\because 14$. & $"$ & 一 & n & tot & -- & tot & 1 & - & tot & - & -- & - \\
\hline$" 15$. & $"$ & - & $n$ & - & - & - & tot & 一 & - & - & - & -- \\
\hline , 16. u. 17. & $"$ & - & $\mathrm{n}$ & - & - & - & - & - & - & - & - & $-\cdots$ \\
\hline$" 18$. & $"$ & - & 1 & - & $\sim$ & 一 & 一 & 一 & - & - & - & - \\
\hline " 19. & $"$ & - & tot & - & - & - & - & 一 & - & - & - & -- \\
\hline
\end{tabular}

1) Die Temperatur schwankte etwa von 0 bis $10^{\circ} \mathrm{C}$, so weit nichts anderes bemerkt wird. 
Auf die ungleiche Lebensdauer der Larven ist hier kein Gewicht zu legen, da die Larven planktonisch gefischt, also verschieden weit in der Resorption des Dottersackes fortgeschritten waren. Die Beendigung der Resorption des Dottersackes führt sicher den Tod herbei, da man nicht imstande ist, Larven von Seefischen geeignet zu füttern.

Das relative bescheidene Ergebnis dieser Versuchsreihe ist also, da vor dem Absterben der Larven in einem Falle Erregung (r), in anderen Lähmung (l) beobachtet wurde. ${ }^{1}$ )

Etwas mehr besagt die Versuchsreihe mit Schollenlarven, Tabelle VII.

Tabelle VII.

Schollenlarven. Beginn des Versuches am 26. Januar 1909.

\begin{tabular}{|c|c|c|c|c|c|c|c|c|c|c|c|c|c|c|c|c|c|c|}
\hline & & 1 & 2 & 3 & 4 & 5 & 6 & 7 & 8 & 9 & 10 & 11 & 12 & 13 & 14 & 15 & 16 & 17 \\
\hline \multirow{3}{*}{\multicolumn{2}{|c|}{$\begin{array}{l}\text { sofortiges Verhalten } \\
\text { nach } 6 \text { Stunden } \\
\text { am 2. Versuchstage }\end{array}$}} & $\mathbf{r}$ & $\mathbf{r}$ & $\mathrm{n}$ & $\mathrm{n}$ & $n$ & $\mathrm{n}$ & $\mathrm{n}$ & $\mathrm{n}$ & $\mathrm{n}$ & $\mathrm{n}$ & $\mathrm{n}$ & $\mathrm{n}$ & $\mathrm{n}$ & $\mathrm{n}$ & $\mathrm{n}$ & $\mathrm{n}$ & $\mathbf{n}$ \\
\hline & & tot & $\mathrm{n}$ & $\mathrm{n}$ & $\mathrm{n}$ & $n$ & $n$ & $\mathrm{n}$ & $n$ & $\mathrm{n}$ & $n$ & $\mathbf{r}$ & $\mathbf{r}$ & $\mathbf{r}$ & tot & tot & tot & tot \\
\hline & & - & $\mathrm{n}$ & $\mathrm{n}$ & $\mathrm{n}$ & $\mathrm{n}$ & $\mathrm{n}$ & $\mathrm{n}$ & $\mathrm{n}$ & n & $\mathrm{n}$ & $\mathrm{n}$ & $\mathrm{n}$ & $n$ & - & - & - & - \\
\hline$" 3$ & $"$ & - & $n$ & $n$ & $n$ & $\mathrm{n}$ & $\mathrm{n}$ & $\mathrm{n}$ & n & $\mathrm{n}$ & tot & $\mathrm{n}$ & $\mathrm{n}$ & tot & - & - & - & - \\
\hline$" 4$. & $"$ & - & $\mathrm{n}$ & $\mathrm{n}$ & $\mathrm{n}$ & $\mathrm{n}$ & $n$ & $\mathrm{n}$ & $\mathrm{n}$ & $\mathrm{n}$ & - & $\mathrm{n}$ & tot & - & - & - & - & - \\
\hline$» 5$ & 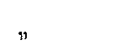 & - & $\mathbf{r}$ & $\mathbf{r}$ & $\mathbf{r}$ & tot & $\mathbf{r}$ & $\mathbf{r}$ & $\mathbf{r}$ & $\mathbf{r}$ & - & $\mathbf{r}$ & - & - & - & - & - & - \\
\hline " 7. & . & - & $\mathbf{r}$ & $\mathbf{r}$ & $\mathbf{r}$ & - & $\mathbf{r}$ & $\mathbf{r}$ & $\mathbf{r}$ & $\mathbf{r}$ & - & tot & - & - & - & - & - & - \\
\hline " 8. & 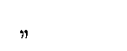 & - & tot & tot & $\mathbf{r}$ & - & $\mathbf{r}$ & $\mathbf{r}$ & $\mathbf{r}$ & $\mathbf{r}$ & - & $\rightarrow$ & - & - & - & - & - & - \\
\hline$" 9$ & . & - & - & - & $\mathbf{r}$ & - & $\mathrm{n}$ & $\mathbf{r}$ & tot & $\mathbf{r}$ & - & - & - & - & - & - & - & - \\
\hline$" 10$ & 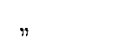 & - & - & - & $\mathbf{r}$ & - & $\mathbf{r}$ & $\mathbf{r}$ & - & $n$ & - & - & - & - & - & - & - & - \\
\hline$" 11$. & $"$ & - & - & - & $\mathbf{r}$ & - & tot & $\mathbf{r}$ & - & 1 & - & - & - & - & - & - & $\cdots$ & - \\
\hline " 12. & $"$ & - & - & - & $\mathrm{n}$ & - & -- & 1 & - & tot & - & - & - & - & - & - & - & - \\
\hline " 13. & $"$ & - & - & - & $\mathrm{n}$ & - & - & 1 & - & - & $\cdots$ & $1-1$ & - & - & - & - & - & - \\
\hline " 14. & " & - & - & - & 1 & - & - & tot & - & - & - & - & - & - & - & - & - & - \\
\hline$" 15$. & $"$ & - & - & - & 1 & - & - & - & - & - & - & - & - & - & - & - & - & - \\
\hline " 16. & $"$ & - & 1 & -- & tot & $1-$ & --1 & 1 & - & - & - & $1-1$ & $1-$ & $|-|$ & $1-$ & - & -1 & - \\
\hline
\end{tabular}

Auch hier ist auf die ungleiche Lebensdauer nicht allzuviel Gewicht zu legen, da die Larven auf dem Stadium des Eies planktonisch gefischt waren.

Immerhin folgt aus dieser Versuchsreihe im verein mit der vorigen und mit den Angaben über Schollenlarven in Salzgehalten zwischen 1 und 2 wohl schon untrüglich, daß sowohl Lösung 1 (destilliertes Wasser), sowie

1) Hs wäre sehr angenehm, wenn den Erregungszuständen bestimmte Zustände der Chromatophoren, etwa Kontraktion derselben, parallel ginge. Leider aber ist dies nicht der Fall, die Chromatophoren scheinen überhaupt in sehr komplizierter Weise zu reagieren, die mir noch nicht klar geworden ist. 
Lösung 10 und stärkere (d. h. Lösungen, die stärker als Nordseewasser sind) lebenabkürzend wirken.

Das ist ja auch nicht wunderbar.

Ich glaube aber, wir können jetzt auch schon einen Schluß ziehen auf die Art und Weise, wie die abnormen Lösungen lebenabkürzend wirken.

Überblickt man nämlich Tabelle VI und VII, so ist die Annahme berechtigt - und sie bestätigt sich im folgenden wiederholt - daß dem Absterben eine Phase der Erregung vorangeht. In ihr glaube ich die Ursache einer zunehmenden zum Tode führenden Ermattung erblicken zu sollen. Auf die Phase der Erregung folgt natürlich noch eine scheinbar normale Phase und eine Phase der Lähmung, denn ohne diose kann os nicht zum Aufhören der Lebenstätigkeit kommen. - In manchen Fällen wurde, da ja pro Tag nur je eine Beobachtung vorliegt, die eine oder die andere Phase nicht beobachtet, trotzdem ist aller Grund vorhanden, anzunehmen, daß sie eingetreten war. Auch ist bei allen diesen Beobachtungen zu bedenken, daß das augenblicklich zu beobachtende Verhalten nicht unbedingt dem für den ganzen Tag durchschnittlichen genau entspricht.

Die Ursache der Erregung kann wenigstens in Lösung 2 bis 7 nicht in einer Wasser- resp. Salzaufnahme resp. Abgabe von seiten des Organismus beruhen, denn hiergegen sprechen entschieden die Ergebnisse über die Osmotik der Fischlarve.

Die Erregung muB also durch den Reiz auf das periphere Nervensystem hervorgerufen sein.

Soweit Erregung und Tod in normalen lösungen eintrat, können wir wohl nur den Reiz auf die sensiblen Magennerven, den Hunger für sie verantwortlich machen. Ich möchte übrigens darauf hinweisen, daß die Versuchsreihe Tabelle VII die einzige ist, wonach auch in Lösung $7(32,7 \%$ \% $\mathbf{r}$ eintrat. Tabelle VI, VIII und IX zeigen dies nicht.

Da aber Erregung und Tod in abnormen Lösungen früher eintreten, so muß hier etwas hinzukommen, und das kann nur der Reiz auf die ehemischen Sinnesorgane sein.

Bei der Versuchsanordnung ist offenbar nicht das Absterben das biologisch Wichtige; es kann aber nun und im folgenden als ganz guter Indikator der voraufgegangenen Erregungsphase aufgefaßt werden; im freien Leben wird das Absterben, falls es überhaupt in Frage kommt (was z. B. in der Ostsee stellenweise sicher anzunehmen ist), wohl meist durch zweckmäßige Wanderungen verhütet werden. Im freien Leben wird also die Erregungsphase das biologisch Wichtige sein.

Erscheint noch manches von den mitgeteilten Beobachtungen wenig zwingend, so erfährt es eine neue Bestätigung durch die folgende Tabelle VIII, welche das Verhalten von Centronotuslarven gegenüber verschiedenem Salzgehalt darstellt. 
Tabelle VIII.

Centronotus-

Beginn des Versuches:

\begin{tabular}{|c|c|c|c|c|c|c|c|c|}
\hline & & 1 & 2 & 3 & 4 & 5 & 6 & 7 \\
\hline \multicolumn{2}{|c|}{ nach 6 Stunden } & tot & $\mathrm{n}$ & $\mathrm{n}$ & $\mathrm{n}$ & $\mathbf{n}$ & $\mathbf{n}$ & $\mathrm{n}$ \\
\hline \multicolumn{2}{|c|}{ am 2. Versuchstage } & - & $\mathrm{n}$ & $\mathrm{n}$ & $\mathrm{n}$ & $\mathrm{n}$ & n & $\mathrm{n}$ \\
\hline \multirow{2}{*}{$" 3}$. & $"$ & - & 1 & $\mathrm{n}$ & $\mathrm{n}$ & $\mathrm{n}$ & $\mathrm{n}$ & $\mathrm{n}$ \\
\hline & & - & $\mathbf{r}$ & $\mathbf{r}$ & $\mathbf{r}$ & $\mathbf{r}$ & $\mathbf{r}$ & $\mathrm{n}$ \\
\hline$" 4$. & $"$ & & & & & & insgesamt & relativ 1 \\
\hline$» 5$. & $"$ & - & 1 und tot & 1 & $\mathbf{r}$ & $\mathbf{r}$ & $\mathrm{n}$ & n \\
\hline$» 6$. & $"$ & - & 1 & $\mathbf{r}$ & $\mathbf{r}$ & $\mathrm{n}$ & $\mathrm{n}$ & $\mathrm{n}$ \\
\hline$" 8$. & " & - & $\mathbf{r}$ & $\mathbf{r}$ & $\mathbf{r}$ & n & $\mathrm{n}$ & $\mathrm{n}$ \\
\hline$" 9$. & $"$ & - & $\mathbf{r}$ & $\mathrm{n}$ & $\mathbf{n}$ & $\mathrm{n}$ & $n$ & $\mathrm{n}$ \\
\hline$" 10$ & ” & - & $\mathbf{r}$ & $\mathrm{n}$ & $\mathrm{n}$ & $\mathrm{n}$ & 11 & viele tot \\
\hline , 11. & $"$ & - & $\mathbf{r}$ & $\mathrm{n}$ & $\mathbf{n}$ & $\mathrm{n}$ & $\mathrm{n}$ & eine tot \\
\hline " 12. & $"$ & - & 1 und tot & 1 und tot & 1 und tot & $\mathrm{n}$ & $\mathrm{n}$ & n \\
\hline$» 13$ & $"$ & 一 & meist tot & meist tot & meist tot & $\underset{\text { einige tot }}{n \text { und }}$ & $\begin{array}{l}n \text { und } \\
\text { einige tot }\end{array}$ & $\mathrm{n}$ \\
\hline " 14. & $"$ & - & tot $\mathrm{u} .11$ & $\mathrm{n}$ & $n$ & $\mathrm{n}$ & $\mathrm{n}$ & $\mathrm{n}$ \\
\hline " 15. & $"$ & - & tot & $\mathrm{n}$ & $\mathrm{n}$ & $\mathrm{n} \mathrm{u} .1$ tot & $\mathrm{n}$ & n \\
\hline "16. u. 17. & " & - & - & tot & $\mathrm{n}$ & $\mathbf{r}$ & r & n \\
\hline " 18. u. 19. & $"$ & - & - & - & tot & $\mathbf{r}$ & $\mathrm{n}$ & $n$ \\
\hline "21. & $"$ & - & - & - & - & tot & I u. 1 tot & n \\
\hline \#22. & $"$ & - & - & - & - & - & 2 tot & n \\
\hline ,23. & $"$ & - & - & - & - & - & $\mathrm{n} 1$ tot & letzte tot \\
\hline , 24 & $"$ & - & - & - & - & - & tot & - \\
\hline
\end{tabular}

Es waren hier in jedes Wasserglas 8 Larven gesetzt worden.

$\mathrm{Da} B$ dem Abstarben ein $\mathbf{r}$-Zustand vorangeht und dieser durch $\mathrm{n}$ und 1 allmählich zum Aufhören des Lebens führt, dem wird auch diese Tabelle in keiner Weise widersprechen; nur in wenigen vereinzelten Fällen wird der Leser Geduld haben müssen mit dem Beobachter, der natürlich $\mathrm{n}$ von $\mathbf{r}$ oder von $\mathbf{I}$ nicht immer sicher unterscheiden konnte und nun seine Beobachtungen, den Notizen genau entsprechend wiedergibt.

Besonders schwach scheint hier übrigens $\mathbf{r}$ eingetreten zu sein in Lösung 7 und 8, d. h. in Salzgehalten von 32,7-38,1\% in Salzgehalten also, die dem Normalen am meisten entsprechen. Es ist dies wohl eine Bestätigung der Annahme, daß abnorme Salzgehalte erregend wirken.

Das Wichtigste, was diese Versuchsreihe (Tabelle VIII) zeigt, ist, daß die Larven auf einen bestimmten Salzgehalt abgestimmt sind in der. Weise, daß in diesem ihre Erregung am geringsten, und daher ihre 
Larven,

Tabelle VIII.

9. Februar 1909.

\begin{tabular}{|c|c|c|c|c|c|c|c|c|}
\hline 8 & 9 & 10 & 11 & 12 & 13 & 15 & 17 & Bemerkungen \\
\hline $\mathrm{n}$ & $\mathrm{n}$ & $\mathrm{n}$ & $\mathbf{n}$ & $\mathrm{n}$ & $\mathrm{n}$ & $\mathrm{n}$ & tot & \\
\hline $\mathrm{n}$ & $n$ & $\mathrm{n}$ & n & $\mathrm{n}$ & meist tot & tot & - & \\
\hline $\mathrm{n}$ & $\mathrm{n}$ & $\mathbf{n}$ & $\mathbf{r}$ & 1 & tot & - & - & \\
\hline $\begin{array}{c}n \\
\text { (infolge }\end{array}$ & $\begin{array}{c}\mathrm{n} \\
\text { Kälte) }\end{array}$ & $\mathbf{r}$ & 1 & tot & - & - & 一 & $\left\{\begin{array}{c}\text { Temperatur }-1^{0}, \\
\text { Glas 1 1. } 2 \text { gefroren } \\
\text { oberflächlich. }\end{array}\right.$ \\
\hline$n$ & $\mathrm{n}$ & $\mathrm{n}$ & $\mathrm{n}$ & - & - & - & - & \\
\hline $\mathrm{n}$ & $\mathrm{n}$ & $\mathrm{n}$ & einige tot & - & - & - & 一 & \\
\hline$n$ & $n$ & $\mathbf{r}$ & tot & - & - & - & 一 & \\
\hline $\mathrm{n}$ & $\mathbf{r}$ & meist tot & - & - & - & - & - & \\
\hline $\mathrm{n}$ & $\begin{array}{c}\text { alle bisauf } \\
1 \text { tot }\end{array}$ & tot & 一 & - & - & 一 & - & \\
\hline $\mathrm{n}$ & $\mathrm{n}$ & 一 & - & - & - & - & - & \\
\hline in & $\mathrm{n}$ & -- & $\cdots$ & - & - & - & - & \\
\hline n & $\mathrm{n}$ & - & - & - & - & - & - & \\
\hline $\mathrm{n}$ & $\mathrm{n}$ & - & - & - & - & - & - & \\
\hline$n$ & $\mathrm{n}$ & - & - & - & - & - & 一 & \\
\hline $\mathrm{n}$ & $\mathrm{n}$ & - & - & - & - & - & - & \\
\hline $\mathrm{n}$ & tot & - & - & - & - & - & - & \\
\hline tot & - & - & - & - & -- & - & - & \\
\hline- & - & - & - & - & - & - & - & \\
\hline- & - & - & - & - & - & - & - & \\
\hline- & - & - & - & - & - & - & - & \\
\hline
\end{tabular}

Lebensdauer im Versuch am größten ist. Es ist dies der Salzgehalt von Lösung 6 und 7, d. h. 27 bis $33 \%$. Dieses Ergebnis kann nur aus diesem Versuch resultieren, weil nur in ihm alle Larven einem Gelege entstammten, mithin gleich alt waren, und weil Zufälle durch eine größere Zahl von Versuchstieren (8) in jedem Glase mehr oder weniger ausgeschaltet wurden. Ubrigens ist ja dieses Ergebnis durchaus dasjenige, welches wir erwarten mußten. Von höherem Werte und von Bedeutung wird es erst durch Verbindung mit nunmehr mitzuteilenden Tatsachen.

Werfen wir zunächst einen Blick auf die Versuchsreihe mit planktonisch gefischten Agonuslarven.

Diese Versuchsreihe spitzt sich hinsichtlich der Lebensdauer zwar recht schön zu, worauf jedoch bei planktonisch gefischten Larven nicht zu viel Wert gelegt werden darf.

Die sofort nach Einsetzen der Larven zu beobachtende Erregung ist 
Tabelle IX.

Agonuslarven.

Beginn des Versuches: 20. Februar 1909.

\begin{tabular}{|c|c|c|c|c|c|c|c|c|c|c|c|c|c|}
\hline & & 1 & 2 & 3 & 4 & 5 & 6 & 7 & 8 & 9 & 10 & 11 & Bemcrkungen \\
\hline \multicolumn{2}{|c|}{ Sofortiges Verhalten } & \begin{tabular}{|c|} 
Sehr \\
$\mathbf{r}$ \\
nach 18 Min. \\
1
\end{tabular} & $\mathbf{r}$ & $\mathbf{r}$ & $\mathbf{r}$ & $\mathbf{r}$ & $\mathbf{r}$ & $\mathbf{r}$ & $\mathbf{r}$ & $\begin{array}{c}\mathbf{1} \\
\operatorname{dann}\end{array}$ & $\mathbf{r}$ & $\mathbf{r}$ & \\
\hline \multicolumn{2}{|c|}{ am 2. Versuchstage } & tot & $n$ & $\mathbf{r}$ & 1 & $n$ & n & $\mathrm{n}$ & $\mathrm{n}$ & $\mathrm{n}$ & $\mathbf{r}$ & $\mathrm{n}$ & \\
\hline$" 3$. & $"$ & - & tot & $\mathrm{n}$ & $\mathrm{n}$ & $\mathrm{n}$ & $n$ & $\mathrm{n}$ & $\mathrm{n}$ & $\mathrm{n}$ & tot & tot & \\
\hline$" 4$ & , & - & $-\infty$ & $\mathrm{n}$ & $\mathrm{n}$ & $n$ & $\mathrm{n}$ & 1 & $\mathrm{n}$ & $\mathrm{n}$ & - & - & \\
\hline$=5$ & , & - & -1 & $\mathrm{n}$ & $\mathrm{n}$ & $\mathrm{n}$ & 1 & 1 & $\mathrm{I}$ & 1 & - & - & \\
\hline 6. u. 7. & $"$ & - & - & $\mathrm{n}$ & $\mathrm{n}$ & $\mathrm{n}$ & $\mathrm{n}$ & 1 & 1 & 1 & - & - & \\
\hline .9 & $"$ & - & - & $\mathbf{r}$ & $\mathbf{r}$ & $\mathbf{r}$ & $\mathbf{r}$ & 1 & $\mathrm{n}$ & 1 & - & - & \\
\hline .10 & , & - & - & $\mathbf{r}$ & $\mathbf{r}$ & $\mathrm{n}$ & $\mathrm{n}$ & 1 & 1 & 1 & - & - & \\
\hline, 11. & . & 一 & - & $\mathbf{r}$ & $\mathbf{r}$ & $\mathbf{r}$ & 1 & $\mathbf{I}$ & 1 & 1 & - & - & \\
\hline .12 & , & - & - & I & $\mathbf{r}$ & $\mathbf{r}$ & I & 1 & 1 & I & -1 & - & \\
\hline., 13. & $"$ & - & - & 1 & 1 & 1 & 1 & I & 1 & tot & - & - & \\
\hline "14. u. 15. & $n$ & - & - & $\mathrm{n}$ & $\mathrm{n}$ & $\mathrm{n}$ & 1 & 1 & 1 & - & - & - & \\
\hline , 17. bis 19 & $"$ & - & - & 1 & 1 & 1 & 1 & I & 1 & - & - & - & \\
\hline, 20 & 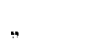 & - & - & 1 & 1 & 1 & tot & tot & tot & - & -- & -- & \\
\hline .23 & $\because$ & - & - & $?$ & tot & $?$ & - & 一 & -- & - & - & - & $\left\{\begin{array}{l}\text { Ich nicht an- } \\
\text { wesend. Zim- }\end{array}\right.$ \\
\hline .24 & $"$ & - & - & 1 & - & $?$ & - & - & - & - & - & - & $\begin{array}{l}\text { mer stark ge- } \\
\text { heizt. Beob- } \\
\text { acht. durch }\end{array}$ \\
\hline . 25 & $"$ & - & - & tot & -- & tot & -1 & - & $\therefore$ & - & $-\|$ & -1 & Diener. \\
\hline
\end{tabular}

wohl auf Rechnung der plötzlich ungewohnten Umgebung zu setzen. Dis Erregung ist bei den stark beweglichen Agonuslarven sehr leicht zu konstatieren, während sie bei den etwas unbeholfeneren Schollenlarven weniger auffiel.

Im übrigen zeigt dieser Versuch aufs neue die dem Tode vorausgehende Erregungsphase an. Sie wurde in Lösung 7 bis 9 (normaler Salzgehalt und etwas stärkerer) nicht beobachtet.

Am 10. Versuchstage habe ich den Erregungszustand der Larven in Glas 3 und 4 mir etwas genauer betrachtet, weil es sehr auffällig war, daß diese Tiere - wie oftmals andere in ähnlichen Fällen - in ununterbrochener, äuBerst lebhafter Bewegung waren. Sollten sio wirklich dauernd in solcher Bewegung verharren? Sollte die Körpermuskulatur also zu einer viel größeren Leistung als gewöhnlich befähigt sein, wenn die Larve mehrere Tage in abnormem Salzgehalte verweilt hat? Durchaus nicht. Nachdem ich die beiden Individuen eine Stunde lang fort- 
gesetzt beobachtet hatte, fiel zuerst das in 3 , dann sehr bald das in 4 auf den Boden, und zwar infolge offenbarer Ermattung auf die Seite.

Wenn also $\mathbf{r}$ beobachtet wurde, so heibt das nicht, daß das Tier zu größerer Energieproduktion befähigt war - wie es vielleicht bei der unwillkürlichen Muskulatur durch erregende Wirkung eintreten kann -, sondern daß es auf schwachen Reiz - Erschütterung - ungemein stark und anhaltend reagierte - darauf freilich auch sehr stark erschöpft war.

Das Tier befindet sich also eigentlich nicht im Zustande danernder Erregung, wie ich es bisher nannte, sondern richtiger gesagt im Zustande einer gesteigerten Frregbarkeit.

Diesen, durch abnorme Sinnesreize hervorgerufenen Zustand möchte ich nicht gerade mit dem wohlbekannten, aber in so allgemeiner Anwendung nicht üblichen Ausdruck Nervosität bezeichnen, sondern nenne ihn lieber mit einem etwas weniger besagenden Worte

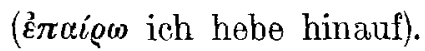

$$
\text { Epäresis }
$$

Welche Bedeutung derselbe hat, werden wir sogleich sehen, wenn wir einige Beobachtungen über den

\section{Phototropismus}

der Fischlarven anfügen.

Weder bei der Schollen-, noch bei der Ammodyteslarve ist der Phototropismus sehr auffällig, obschon er bei beiden vorhanden ist: positiv bei diffusem Tageslichte, negativ (bei der Schollenlarve) in direktem Sonnenlichte ${ }^{1}$ ). Sehr viel auffälliger ist dagegen der Phototropismus bei den benthopelagischen Larven von Centronotus und von Agonus. (Über Cottus habe ich keine hierher gehörigen Beobachtungen.) In einem Glase, welches man vor ein nach Norden gelegenes Fenster stellt, lassen die beiden Larven eine sehr starke, positiv-phototropische Reaktion erkennen

Ich habe nun einige Beobachtungen mitzuteilen, die vielleicht geeignet sind, auf das Wesen des Phototropismus überhaupt ein neues Licht zu werfen:

1. Bei Centronotus-, wie bei Agonuslarven habe ich beobachtet, daß sie, in einem hinreichend großen Behälter vollkommen sich selbst überlassen, ihren Phototropismus so zu sagen vergaßen, d. h. nach 24 Stunden zeigten sie keine Orientierung nach den Lichtstrahlen mehr, sondern schwammen meist nahe dem Boden hierhin und dorthin, so lange man sich ihnen ganz vorsichtig näherte.

1) Diese sehr allgemein gehaltene Angabe soll nichts darüber aussagen, ob der Phototropismus durch längere Einwirkung des Lichtreizes den Sinn wechseln kann, wie Loeb bei Balanuslarven annimmt. 
2. Die geringste Erschütterung aber bewirkte, daß die Tiere sämtlich der Lichtseite des Behälters zueilten. Man hatte den Eindruck, daß sie das Nahen einer Gefahr fühlten und nach dem Lichte hin flohen.

3. Der Phototropismus ist stets um so ausgeprägter, je mehr Individuen in einem Wasserglase mit $100 \mathrm{ccm}$ Inhalt vorhanden sind. Die genaue Beobachtung der einzelnen Tiere hat mich zu der festen Uberzeugung geführt, daß in einem dichter besetzten Glase jedes einzelne Tier stärker phototropisch ist, als im schwächer besetzten, und daß nicht etwa die größere Gesamtheit aller Tiere dieses nur vortäuscht.

Das Gemeinsame von Punkt 1 bis 3 können wir darin zusammenfassen, daß alles Abnorme in den Lebensbedingungen den Phototropismus erhöht, oder auch: daß im Zustande der Erregtheit der Phototropismus erhöht ist.

Und dazu paßt nun vorzüglich die oftmals von mir gemachte Beobachtung,

4. daB auch in abnormem Salzgehalt, also im Zustande der Epäresis, der Phototropismus erhöht ist. Tatsächlich zeigte sich bei Centronotus- und Agonus-Larven überall da die phototropische Reaktion am deutlichsten, wo $\mathbf{r}$ konstatiert wurde, oder anders ausgedrückt: die konstatierte Erregung war immer eine phototropische Erregung.

Es ist wohl kaum zu leugnen, daß diese Beobachtungen den Phototropismus in einem anderen Lichte als bisher erscheinen lassen, wenigstens bei den Fischlarven - bei denen er allerdings bisher noch nicht beobachtet oder studiert wurde.

Es ist zunächst der Schluß ganz unumgänglich, daß die phototropische Reaktion im Laboratoriumsversuch oft viel stärker ausfällt, als im freien Leben.

Sodann ist die Bedeutung der phototropischen Reaktion aus diesen Beobachtungen vielleicht etwas richtiger als aus allen bisherigen zu erraten.

Wenn nämlich der (positive) Phototropismus sich immer am stärksten bei den im augenblicklichen Erregungszustande befindlichen Larven zeigt, so erscheint er gewissermaßen als ein "das Weite suchen" vor nahender Gefahr oder vor Unbehaglichkeit. Er erscheint also als eine höchst komplizierte Gewohnheit und vielleicht als eine zweckmäßige Funktion. Denn wenigstens eine benthopelagische Fischlarve hat, wenn sie das Weite, das offene Meer sucht, viel größere Chancen, einer Gefahr zu entgehen, als wenn sie planlos flieht.

Wie mag es zustande kommen, daB die Tiere, die durch eine richtungslose Ursache, wie ungewohnte Umgebung, allgemeine Erschütterung, Gedränge, in einen Erregungszustand versetzt werden (der nach längerem Aufenthalt in abnormem Salzgehalt, infolge Epäresis, noch leichter eintritt), 
nun auf den gerichteten Reiz, das Licht, so besonders stark reagieren? Die Sache liegt vielleicht so: Ohne richtende Ursache kann keine den Organismus befriedigende Reaktion eintreten, weil die Reaktion, die Körperbewegung, an sich immer irgend eine Richtung hat, für den Organismus aber kein Grund vorliegt, eine Richtung zu bevorzugen. Mag also bei fehlendem Lichtreiz auch eine Reaktion zustande kommen, es wird ein gewisser Teil der Impulse unausgelöst bleiben: sie werden, gleichviel ob sie unterschwellig sind oder nicht, in gewissem Sinne eine "Bahnung“ (Exner) im ganzen Nervensystem herbeiführen. Zur starken Reaktion kommt es dann begreiflicherweise nur in den Teilen des Körpers, in welchen sich die vom richtenden Reize, dem Lichte, kommenden Impulse zu den schon vorhandenen addieren. Im Grunde ist die Frage wohl fast ebenso schwierig wie die, weshalb ein erregter oder ein nervöser - erregbarer Mensch eine gröBere Großstadtflucht an den Tag legt als ein normaler.

SchieBlich ist es augenblicklich nicht so wichtig, die Verstärkung der phototropischen Reaktion unter abnormen Bedingungen zu erklären, als sie zu beschreiben, d.h. die Entdeckung dieser Erscheinung festzustellen. Denn sie kann vielleicht zur Erklärung größerer, noch komplizierterer Phänomene dienen. Erklären heißt analysieren.

Suchen wir also jetzt einen Ausblick nach unserem Ziele zu gewinnen, nach der Frage: Wie können die Bedingungen des Salzgehaltes maßgebend sein für die Wanderungen von Fischlarven? Die Antwort läßt sich noch nicht geben, jedenfalls aber hat sich gezeigt, daß gewisse Abweichungen von einem normalen Salzgehalt nicht nur erregend wirken, sondern auch den Phototropismus verstärken. 